\title{
Asociación entre recuento bacteriano en la bilis y desarrollo de morbilidad postoperatoria en pacientes con colangitis aguda*
}

\author{
Drs. HÉCTOR LOSADA M. ${ }^{1,2}$, CARLOS MANTEROLA D. ${ }^{1,2}$, VIVIANA PINEDA N.., ${ }^{1,2}$, \\ MANUEL VIAL G. ${ }^{1,2}$, LUIS AVENDAÑO R. ${ }^{1}$, \\ GRUPO MINCIR. \\ Departamento de Cirugía y Traumatología, Universidad de La Frontera. \\ 2 Servicio de Cirugía. Hospital Hernán Henríquez Aravena, Temuco, Chile.
}

\begin{abstract}
\section{Association between bile bacterial count and postoperative complications among patients with acute cholangitis}

Background: Bacterial colonization of bile is common in patients with bile duct obstruction, even in the absence of clinical signs of sepsis. Aim: To determine the association between bile bacterial count and postoperative complications in patients with bile duct obstruction. Material and Methods: A bile bacterial count was performed to patients with acute cholangitis caused by biliary stones, operated between 2004 and 2006. Post operative complications and length of hospital stay were recorded in these patients. Results: Fifty four patients aged between 25 and 95 years (34 females) were studied. The most common bacteria found, were Escherichia coli in 32 cultures (50\%), followed by Klebsiella pneumoniae in 6 cultures (9\%). In 45 cases the bacterial load was over $10^{5}$ colony forming units $(\mathrm{CFU}) / \mathrm{ml}$. Among patients with and without complications, 68 and $69 \%$ had a bacterial load over $10^{5} \mathrm{CFU} / \mathrm{ml}$, respectively $(\mathrm{p}=\mathrm{NS})$. A multivariate analysis showed an association between platelet count and prothrombin time with the incidence of complications. Conclusions: In this series of patients with acute cholangitis, no association between bile bacterial count and postoperative complications was observed.

Key words: Bile culture, cholangitis, postoperative complications.

\section{Resumen}

Introducción: La colonización bacteriana de la bilis parece ser frecuente en pacientes con obstrucción de la vía biliar ya sea con o sin signos de sepsis. Objetivos: Determinar asociación entre el recuento bacteriano en la bilis de pacientes con colangitis aguda (CA) y el desarrollo de complicaciones postoperatorias; y, determinar asociación entre variables fisiológicas y desarrollo de complicaciones en el mismo grupo. Material y Método: Reporte preliminar de estudio de cohorte de pacientes con CA litiásica, intervenidos quirúrgicamente en los servicios de cirugía y urgencias del Hospital Regional de Temuco en el

\footnotetext{
*Recibido el 10 de Abril y aceptado para publicación el 7 de Agosto de 2008.

Financiado por proyecto DIDUFRO INI N 110408.

Correspondencia: Dr. Héctor Losada $\mathrm{M}$.

Casilla 54-D. Temuco, Chile.

E-mail: hlosada@ufro.cl
} 
período diciembre de 2004 a noviembre de 2006. Se realizó cultivo cuantitativo y se registraron variables clínicas y de laboratorio al ingreso. Se efectuó medición de complicaciones postoperatorias, estancia hospitalaria y evolución de los pacientes. Se aplicó estadística descriptiva y posteriormente análisis univariado y multivariado. Resultados: Se estudió un total de 54 pacientes, 34 (63\%) fueron de género femenino y 20 (37\%) de género masculino, con una mediana de edad de 68 años (25 a 95 años). El germen más frecuentemente aislado fue Escherichia coli en 32 cultivos (50\%), seguido por Klebsiella pneumoniae en 6 cultivos (9\%). De los gérmenes aislados 45 (82\%) tenían cargas bacterianas sobre $10^{5} \mathrm{UFC} / \mathrm{ml}$. De los pacientes que presentaron alguna complicación $68 \%$ tenían cargas bacterianas superiores a $10^{5} \mathrm{UFC} / \mathrm{ml}$ y de los pacientes que no presentaron complicaciones $69 \%$ tenían cargas bacterianas inferiores a $10^{5} \mathrm{UFC} / \mathrm{ml}(\mathrm{p}=0,513)$. En el análisis univariado se encontró asociación entre desarrollo de complicaciones y las variables frecuencia cardíaca, creatinina, recuento de plaquetas y tiempo de protrombina. Con el análisis multivariado se determinó asociación entre desarrollo de complicaciones y las variables recuento de plaquetas y tiempo de protrombina (OR: 4,35 y 4,58). Conclusiones: No se encontró asociación entre la carga bacteriana y el desarrollo de complicaciones en pacientes con CA. Se determinó asociación entre desarrollo de complicaciones y las variables recuento de plaquetas y tiempo de protrombina.

Palabras clave: Colangitis, cultivos cuantitativos de bilis, estudio de cohorte.

\section{Introducción}

La colangitis aguda (CA), es una entidad prevalente en Chile, sin embargo, el modelo fisiopatólogico, incluido el rol de la carga bacteriana ha sido poco estudiado ${ }^{1}$.

La colonización bacteriana de la bilis parece ser frecuente en pacientes con obstrucción de la vía biliar con o sin signos de sepsis; sin embargo, no se ha establecido el rol de la carga de bacterias en la fisiopatología o evolución de los pacientes con $\mathrm{CA}^{2}$.

En un reporte previo se encontró que en pacientes con manifestaciones clínicas de CA, la carga bacteriana era superior a $10^{5}$ Unidades Formadoras de Colonias (UFC) $/ \mathrm{mm}^{3}$; lo que contrasta con la experiencia de nuestro equipo de trabajo, pues verificamos que en pacientes con CA la carga bacteriana era mayor a $10^{5} \mathrm{UFC} / \mathrm{ml}$, sólo en el $83 \%$ de los casos ${ }^{4}$.

La hipótesis de este estudio es que existe asociación entre el recuento bacteriano en la bilis de pacientes con CA sometidos a drenaje de la vía biliar por cirugía abierta y el desarrollo de complicaciones postoperatorias.

Los objetivos del presente trabajo son: Determinar asociación entre el recuento bacteriano en la bilis de pacientes con CA litiásica sometidos a drenaje de la vía biliar por cirugía abierta y el desarrollo de complicaciones postoperatorias; determinar asociación entre variables fisiológicas y el desarrollo de complicaciones postoperatorias; y, describir la carga bacteriana y gérmenes encontrados en la bilis coledociana de pacientes con CA litiásica.

\section{Material y Método}

Diseño: Estudio de cohorte prospectiva.
Población: Se incluyeron sujetos mayores de 18 años con colangitis aguda litiásica (definida por tríada de Charcot y/o parámetros de sepsis de origen biliar presentes al ingreso al servicio de urgencias), intervenidos quirúrgicamente con drenaje abierto de vía biliar, con o sin colecistectomía, en los servicios de cirugía y urgencias del Hospital Regional de Temuco en el período diciembre de 2004 a noviembre de 2006 y que aceptaron participar de éste protocolo mediante la firma del consentimiento informado. Se excluyeron, pacientes con sospecha de neoplasia vesicular, de la vía biliar o periampular; usuarios de corticoesteroides o fármacos inmunodepresores hasta 15 días antes de la consulta por el cuadro abdominal; pacientes que hubiesen recibido antibióticos 48 horas previo a la consulta por el cuadro abdominal; y pacientes a los cuales se les realizó derivación biliodigestiva o cierre primario de la vía biliar sin coledocostomía.

Variables: La variable de exposición fue el cultivo con carga bacteriana mayor a $10^{5} \mathrm{UFC} / \mathrm{mm}^{3}$; y la variable de desenlace, el desarrollo de complicaciones postoperatorias. Se utilizaron como variables de control, variables clínicas (edad, género, frecuencia cardíaca, frecuencia respiratoria y temperatura axilar al ingreso); variables de laboratorio (hematocrito, recuento total de leucocitos, fosfatasa alcalina, transaminasas, creatinina y tiempo de protrombina); variables microbiológicas (gérmenes aislados, sensibilidad y resistencia antibiótica y recuento bacteriano, este última categorizada en mayor y menor a $10^{5} \mathrm{UFC} / \mathrm{ml}$ ); variables de la evolución clínica (tiempo de hospitalización, desarrollo de complicaciones postoperatorias como sepsis abdominal, neumonía, infección asociada a catéter central, infección urinaria, tromboembolismo pulmonar, infarto agudo de miocardio; y mortalidad). 
Maniobra: Una vez identificada la vía biliar, se realizó punción con aguja previa a la coledocotomía y se procedió a sembrar inmediatamente los cultivos en agar sangre y agar Mac Conkey. La sensibilidad in vitro a los antibióticos se realiza por medio de método cualitativo con sensidiscos para cada tipo de antibiótico.

Tamaño de la muestra: No se encontró ningún reporte en la literatura donde se haya estudiado la asociación entre la carga bacteriana de la bilis de los pacientes con CA y las complicaciones postoperatorias, razón por la que no contamos con ninguna estimación para el cálculo de la muestra necesaria. Por la razón antes expuesta, es que decidimos hacer el primer reporte de nuestra cohorte al completar 54 pacientes.

\section{Definiciones:}

Triada de Charcot. Coexistencia de los siguientes signos y síntomas: Temperatura axilar o rectal mayor a $38^{\circ} \mathrm{C}$, dolor a la palpación superficial en hipocondrio derecho, ictericia en piel o escleras ${ }^{5}$.

Sepsis de origen biliar. Presencia de 2 ó más de los siguientes signos: Temperatura axilar de $38^{\circ} \mathrm{C}$ o $36^{\circ} \mathrm{C}$; frecuencia cardíaca $\geq 90$ latidos $/ \mathrm{min}$; frecuencia respiratoria $\geq 20$ respiraciones $/ \mathrm{min}$ o $\mathrm{PaCO}_{2}$ de 32 torr; recuento de leucocitos $\geq 12.000$ células $/ \mathrm{mm}^{3}$, $\leq 4.000$ células $/ \mathrm{mm}^{3}, \mathrm{o} \geq 10 \%$ de formas inmaduras. Esto asociado siempre a la evidencia ultrasonográfica de dilatación de la vía biliar intrahepática o extrahepática, ésta última considerada como colédoco mayor o igual de $7 \mathrm{~mm}$ en cualquier proyección.

Unidad Formadora de Colonias (UFC). Célula viva y aislada que se encuentra en un sustrato y en condiciones ambientales adecuadas, que produce una colonia en un lapso de tiempo breve.

Plan de análisis: Los datos fueron analizados utilizando el programa Stata ${ }^{\circledR}$ 8.0. Se utilizó estadística descriptiva, con cálculo de medidas de tendencia central, dispersión y tendencia extrema; análisis divariado utilizando chi $^{2}$ de Pearson o test exacto de Fischer para variables categóricas y t-test, ANOVA y Kruskal Wallis para variables continuas. Posteriormente, se aplicaron modelos de regresión logística para la estimación de la magnitud de la asociación, calculando OR e intervalos de confianza del $95 \%$.

Aspectos Éticos y de financiamiento: Este protocolo fue aprobado por el Comité de Ética de la Facultad de Medicina de la Universidad de La Frontera y financiado por el proyecto DIDUFRO INI ${ }^{\circ}$ 110408 de la Dirección de Investigación y Desarrollo de la Universidad de La Frontera.

\section{Resultados}

Se estudió un total de 54 pacientes, los que presentaron una mediana de edad de 68 años $(25$ a 95 años) y $63 \%$ de género femenino.

Cuarenta y un pacientes (76\%), presentaron triada de Charcot al ingreso. Las variables clínicas de ingreso y los parámetros de laboratorio más importantes se describen en la Tabla 1.

Treinta y cinco cultivos de bilis fueron monobacterianos $(64,8 \%), 10$ polibacterianos $(18,5 \%)$; lo que resulta en un total de 64 gérmenes aislados; y 9 cultivos fueron negativos $(16,7 \%)$.

De los gérmenes aislados, 50 (78\%) tenían cargas bacterianas sobre $10^{5} \mathrm{UFC} / \mathrm{ml}, 4(22 \%)$ presentaron carga bacteriana inferior a $10^{5} \mathrm{UFC} / \mathrm{ml}$. El germen más frecuentemente aislado fue Escherichia coli en 32 cultivos (50\%), seguido por Klebsiella pneumoniae en 6 cultivos (9\%). Citrobacter freundii, Pseudomona aeruginosa y Morganella morganii se aislaron en 2 cultivos respectivamente y se aisló levaduras en 1 cultivo.

$\mathrm{Al}$ observar la resistencia de los gérmenes a los antibióticos de uso habitual en nuestro centro, destacamos que en 2 cultivos Escherichia coli y Klebsiella pneumoniae y en 1 cultivo Morganela morganii se registró resistencia a cefalosporinas de tercera generación y quinolonas. Enterococo registró 1 caso de resistencia a ampicilina.

Tabla 1. Variables clínicas y de laboratorio al ingreso

\begin{tabular}{lcc}
\hline Variables & Mediana & $\begin{array}{c}\text { Valores } \\
\text { extremos }\end{array}$ \\
\hline Frec cardíaca (lat/min) & 86 & $60-132$ \\
Temperatura axilar $\left({ }^{\circ} \mathrm{C}\right)$ & 38 & $6-40,5$ \\
PAS (mmHg) & 127,5 & $71-170$ \\
PAD (mmHg) & 74,5 & $43-100$ \\
Leucocitos (cel/ml) & 12800 & $3330-53400$ \\
Plaquetas (cel/ml) & 207000 & $23000-879000$ \\
PT $(\%)$ & 71,1 & $19,8-89$ \\
Creatinina (mg/dl) & 1 & $0,6-4,45$ \\
Fosfatasas alcalinas (UI/l) & 651 & $99-4450$ \\
Bilirrubina total (mg/dl) & 5,6 & $0,9-17,7$ \\
Bilirrubina directa (mg/dl) & 3,6 & $0,2-9,3$ \\
\hline
\end{tabular}

Frec cardíaca: frecuencia cardíaca.

PAS: presión arterial sistólica.

PAD: presión arterial diastólica.

PT: tiempo de protrombina. 
Tabla 2. Análisis univariado de variables asociadas con complicaciones

\begin{tabular}{lccc}
\hline Variable & $\begin{array}{c}\text { Sin complicación } \\
(\mathbf{n}=\mathbf{3 5})\end{array}$ & $\begin{array}{c}\text { Con complicación } \\
(\mathbf{n}=\mathbf{1 9})\end{array}$ & $\mathbf{p}$ \\
\hline Edad (años) & $64,9 \pm 15,8$ & $68,6 \pm 13,5$ & 0,39 \\
Temperatura axilar $\left({ }^{\circ} \mathrm{C}\right)$ & $37,8 \pm 0,9$ & $38,3 \pm 1,3$ & 0,10 \\
Recuento de leucocitos (células/mm $\left.{ }^{3}\right)$ & $14.623,4 \pm 9.018$ & $13483,2 \pm 9026$ & 0,65 \\
Bilirrubina total (mg/dl) & $5,8 \pm 2,9$ & $6,9 \pm 3,6$ & 0,18 \\
Frecuencia cardíaca (latidos/min) & $85,5 \pm 14,9$ & $99,6 \pm 21,9$ & 0,0191 \\
Recuento de plaquetas (células/mm $\left.{ }^{3}\right)$ & $270.088,2 \pm 148.366,5$ & $164568,4 \pm 122002,1$ & 0,0110 \\
Protrombina (\%) & $76,1 \pm 12,9$ & $63,1 \pm 20,6$ & 0,0232 \\
Creatinina (mg/dl) & $1,1 \pm 0,5$ & $1,6 \pm 0,9$ & 0,0374 \\
\hline
\end{tabular}

Tabla 3. Análisis multivariado de variables asociadas con complicaciones

\begin{tabular}{lccc}
\hline Variable & $\mathbf{O . R}$ & $\mathbf{p}$ & IC (95\%) \\
\hline Frecuencia cardíaca (latidos/min) & 2,46 & 0,286 & $0,47-12,91$ \\
Recuento de plaquetas (células/mm $\left.\mathrm{mm}^{3}\right)$ & 4,35 & 0,046 & $1,02-18,50$ \\
Creatinina (mg/dl) & 1,75 & 0,443 & $0,41-7,31$ \\
Protrombina (\%) & 4,58 & 0,047 & $1,01-20,66$ \\
\hline
\end{tabular}

La morbilidad de la serie fue de 35,2\% (19 pacientes) y la mortalidad de la serie fue de 5,6\% (3 pacientes). La complicaciones más frecuentes fueron sepsis abdominal (15 casos, $27,8 \%$ ), neumonía ( 9 casos, 16,7\%) e infección asociada a catéter central en ( 3 casos, 5,6\%). El 68\% de los pacientes que presentaron alguna complicación tenían una carga bacteriana superior a $10^{5} \mathrm{UFC} / \mathrm{ml}$; y, el $69 \%$ de aquellos que no desarrollaron morbilidad, tenían una carga bacteriana superior a $10^{5} \mathrm{UFC} / \mathrm{ml}(\mathrm{p}=0,513)$.

En el análisis univariado se encontró asociación entre desarrollo de complicaciones y las variables frecuencia cardíaca, creatinina, recuento de plaquetas y tiempo de protrombina (Tabla 2). En el análisis multivariado se determinó asociación entre desarrollo de complicaciones y las variables recuento de plaquetas y tiempo de protrombina (OR de 4,4 y 4,6 respectivamente) (Tabla 3 ).

El promedio de estancia en terapia intensiva fue de $0,7 \pm 1,8$ días y el promedio de estancia en terapia intermedia fue de $2,8 \pm 5,8$ días. El promedio total del tiempo de hospitalización fue de 14,0 \pm 35,9 días. La mediana del tiempo de hospitalización para los pacientes que no presentaron complicaciones fue de 4,5 días y la mediana de hospitalización de los pacientes con complicaciones fue de 13,5 días.

\section{Discusión}

El cuadro clínico de CA y sepsis de origen biliar parece ser más complejo que la sola teoría de la colonización bacteriana de la vía biliar con reflujo colangiovenoso de gérmenes y mediadores inflamatorios.

La colonización bacteriana es un evento importante, pero el mecanismo a través del cual se producen las manifestaciones clínicas de CA a partir de esta colonización y proliferación no queda claro. Hay reportes de positividad de cultivos en pacientes con coledocolitiasis sin signos de CA que oscilan entre $36 \%$ y $66 \% \%^{6,7}$, hay pocos reportes de hemocultivos en pacientes con CA, mostrando una positividad de hemocultivos de $55 \%^{8}$, datos que no permiten precisar el mecanismo de paso de bacterias directamente de la vía biliar al hígado y sangre periférica.

En nuestro estudio destaca la cifra de 9 cultivos negativos $(16,6 \%)$, y al respecto, se ha de recalcar que la bilis fue sembrada durante el acto quirúrgico. Esta situación podría deberse a que no se consideró el cultivo para anaerobios debido a problemas técnicos del laboratorio, hecho que asumimos como una de las limitaciones del estudio.

Continuando con la idea de cavilar acerca de la 
fisiopatología de la CA, parecería lógico que a mayor carga bacteriana en la bilis, mayor reflujo colangiovenoso existiese, lo que generaría una mayor gravedad del cuadro séptico y posibilidad de complicaciones. Sin embargo, nuestro estudio muestra que no existe asociación entre carga bacteriana y el desarrollo de las complicaciones medidas. Nos parece que el considerar sólo la variable "carga bacteriana", deja de lado la respuesta del sistema inmune del huésped al cuadro inicial y las consecuencias sobre la función de los diversos sistemas. Esto queda un poco insinuado en las variables que se asociaron al desarrollo de complicaciones, como el recuento de plaquetas y el tiempo de protrombina.

Otra de las limitaciones del estudio es el no haber podido calcular el tamaño de la muestra necesario para contar con una potencia apropiada al momento de generar la discusión y las conclusiones; por ello es que el análisis univariado y multivariado se presenta sólo como análisis preliminar, y la idea es continuar incrementando la muestra para darle mayor validez interna a nuestros resultados.

En los análisis de asociación con el desarrollo de complicaciones destaca la asociación en el análisis univariado de variables fisiológicas como creatinina, recuento de plaquetas, tiempo de protrombina y frecuencia cardíaca; y en el análisis multivariado destaca la asociación de las variables del sistema hematológico: plaquetas $\left(\mathrm{OR} 4,35 ; \mathrm{IC}_{95}\right.$ : $1,02-18,50)$ y tiempo de protrombina $\left(\mathrm{OR} 4,58 ; \mathrm{IC}_{95}\right.$ : 1,01-20,66). Todo esto parece estar más en el contexto de un cuadro de sepsis originado en la vía biliar, donde la repercusión sobre la función de sistemas juega un papel preponderante en la morbilidad y mortalidad ${ }^{9-11}$. La creatinina y la frecuencia cardíaca hacen parte del escore de APACHE II; la creatinina y el recuento de leucocitos del escore de SOFA y el tiempo de protrombina y las plaquetas en el escore de coagulación intravascular diseminada $^{12,13}$. No hay estudios con buen diseño que evalúen estos escore en la asociación y predicción de complicaciones y mortalidad en éste grupo de pacientes, lo cual será una de nuestras futuras líneas de investigación.

En concordancia con esto, creemos que una temprana y adecuada resucitación, con una terapia de soporte de sistemas y terapia antibiótica es indispensable en éste grupo de pacientes acompañado del drenaje de la vía biliar.

En relación a la bacteriología, encontramos similitud con otros estudios en cuanto a los gérmenes aislados con mayor frecuencia (Escherichia coli y Klebsiella pneumoniae ${ }^{14,15}$. Sin embargo, llama la atención la presencia de desarrollo de gérmenes nosocomiales como Pseudomona aeruginosa, Citrobacter freundii, Morganella morganii y le- vaduras. En comparación con nuestro reporte previo llama la atención el aumento de resistencia a las cefalosporinas y quinolonas de los gérmenes gram negativos. También llama la atención de la existencia de 1 caso de enterococo resistente a ampicilina.

Las cifras morbilidad y mortalidad de nuestra cohorte son comparables con cifras internacionales ${ }^{16}$.

A modo de conclusión, podemos señalar que no se encontró asociación entre la carga bacteriana y el desarrollo de complicaciones en pacientes con CA; se encontró asociación entre el desarrollo de complicaciones y las variables recuento de plaquetas y tiempo de protrombina; los gérmenes más frecuentemente encontrados fueron similares a estudios previos; y, que la mayor parte de los gérmenes aislados presentaban cargas bacterianas superiores a $10^{5} \mathrm{UFC} / \mathrm{ml}$.

\section{Referencias}

1. Sung JY, Costeron JW, Shaffer EA. Defense system in the biliary tract against bacterial infection. Dig Dis Sci 1992; 37: 689-696.

2. Harris HW. Biliary Sistem. Surgery. Basic and Clinical Evidency. New York: Norton JA, Bollinger RR, Chang AE, Lowry SF, Mulvihill SJ, Pass HI, et al, 2001: 553-584.

3. Flores C, Maguilnik I, Hadlich E, Goldani LZ. Microbiology of choledochal bile in patients with choledocholithiasis admitted a tertiary hospital. J Gastroenterol Hepatol 2003; 18: 333-336.

4. Losada H, Manterola C, Pineda V, Vial M. Recuento bacteriano en bilis de pacientes con colangitis aguda. Reporte preliminar. Rev Chil Cir 2006; 58: 35-39.

5. Charcot JM. Lecons sur les du fore des voices filares et des vans, thesis. Paris 1877.

6. Chang WT, Lee KT, Wang SR, Chuang SC, Kuo KK, Chen JS, et al. Bacteriology and antimicrobial susceptibility in biliary tract disease: an audit of 10years experience. Kaohsiung J Med Sci 2002; 18: 221 228.

7. Csendes A, Mitru N, Maluenda F, Díaz JC, Burdiles P, Csendes $\mathrm{P}$, et al. Counts of bacteria and pyocites of choledochal bile in controls and in patients with gallstones or common bile duct stones with or without acute cholangitis. Hepatogastroenterology 1996; 43 : 800-806.

8. Prevot L, Bresler L, Muller C, Boissel P, Grosdidier J. Bacteriological aspects of acute cholangitis with gallstones. Presse Med 1991; 20: 689-691.

9. Awad SS. State-of-the-art therapy for severe sepsis and multisystem organ dysfunction. Am J Surg 2003; 186 (5A): 23S-34S.

10. Shapiro N, Howell MD, Bates DW, Angus DC, Ngo L, 
Talmor D. The association of sepsis syndrome and organ dysfunction with mortality in emergency department patients with suspected infection. Ann Emerg Med 2006; 48: 583-590.

11. Losada H, Manterola C, Vial M, Pineda V. Sepsis de origen biliar ¿Alternativa diagnóstica en pacientes con colangitis aguda litiásica? Rev Chil Cir 2004; 56: 562566.

12. Losada H, Manterola C, Vial M, Pineda V. SRIS, sepsis y SDOM: una visión quirúrgica. Rev Chil Cir 2004; 56: $373-379$.

13. Kinasewitz GT, Zein JG, Lee GL, Nazir SA, Taylor FB Jr. Prognostic value of a simple evolving disseminated intravascular coagulation score in patients with severe sepsis. Crit Care Med 2005; 33: 2417-2418.

14. Mukaiya M, Hirata K, Katsuramaki T, Kihara C, Kimura Y, Yamaguchi K, et al. Isolated bacteria and susceptibilities to antimicrobial agents in biliary infections. Hepatogastroenterology 2005; 52: 686-690.

15. Maluenda F, Csendes A, Burdiles P, Díaz J. Bacteriological study of choledochal bile in patients with common bile duct stones, with or without acute suppurative cholangitis. Hepatogastroenterology 1989; 36: 132-135.

16. Kumar R, Sharma BC, Singh J, Sarin SK. Endoscopic biliary drainage for severe acute cholangitis in biliary obstruction as a result of malignant and benign diseases. Hepatogastroenterology 2004; 19: 994-997. 